\title{
Biodegradable Materials for Medical Applications II
}

\author{
JAROSLAW W. DRELICH ${ }^{1,},{ }^{1,6}$ MALGORZATA SIKORA-JASINSKA, ${ }^{1}$ \\ EHSAN MOSTAED ${ }^{1}$ HUINAN LIU ${ }^{2}$ PETRA MAIER,${ }^{3}$ \\ JAN-MARTEN SEITZ, ${ }^{4}$ and NORBERT HORT ${ }^{5}$
}

\begin{abstract}
1.-Department of Materials Science and Engineering, Michigan Technological University, Houghton, MI 49931, USA. 2.-Department of Bioengineering, University of California-Riverside, Riverside, CA 92521, USA. 3.-University of Applied Sciences Stralsund, 18435 Stralsund, Germany. 4.-Syntellix AG, 30159 Hannover, Germany. 5.-Helmholtz-Zentrum Geesthacht, 21502 Geesthacht, Germany. 6.-e-mail: jwdrelic@mtu.edu
\end{abstract}

To mitigate the long-term side effects associated with current corrosion-resistant implants, a new generation of bioabsorbable medical devices is currently being developed and have already been approved in some markets (e.g., Europe). Implants made of biodegradable materials are absorbed and excreted by the body after completing their temporary mechanical, scaffolding, and biointegration functions. Biochemical and mechanical attributes of all classes of materials, including metals, ceramics, and polymers, have been broadly explored by scientific and industrial research and development laboratories for various clinical applications over the last 2 decades. The second (biannual) international symposium Biodegradable Materials for Medical Applications took place during the TMS 2020 Annual Meeting in San Diego, CA and addressed the emerging multidisciplinary field of biodegradable materials and implants, involving materials scientists and engineers working with biologists, bioengineers, and medical personnel. The symposium had four oral sessions with 4 keynote presentations, 7 invited talks, and 18 regular presentations, and a poster session with 11 posters. Papers presented covered a broad range of topics related to materials selection, development, processing, and testing, material surface treatments, and modifications, in vitro/in vivo performance assessment, and evaluation of biodegradable-based implants, including vascular, orthopedic, tissue engineering, and other applications, presented by representatives from Canada, China, Germany, Hong Kong, Italy, Poland, Singapore, Slovenia, and the USA. Nearly two dozen selected quality papers were submitted for publication into three

Jaroslaw Drelich, Malgorzata Sikora-Jasinska, Ehsan Mostaed, Huinan Liu, Petra Maier, Jan-Marten Seitz, and Norbert Hort are guest editors for the topic Biodegradable Materials for Medical Applications II. journals, including JOM (this issue), Metallurgical and Materials Transactions $A$ (to be published in volume 51), and Surface Innovations (to be published in volume 8). Although the papers from the first symposium in this series were not published, last year, the April 2019 issue of JOM (vol. 71, no. 4) offered five papers on characterization of biodegradable medical materials. The papers can be downloaded from the journal website, with the table of contents page for this issue available at: http://link. springer.com/journal/11837/71/4/page/1.

Biodegradable polymers are already broadly used in the medical field because of their predictable degradation products, acceptable biocompatibility, and identical degradation mechanisms in vitro and in vivo. In the paper "Bio-absorbable Cardiovascular Implants: Status and Prognosis," Subbu Venkatraman and his colleagues from Singapore present a review on the use of polymer-based devices in various cardiovascular applications, including coronary and peripheral stents, occluders for heart defects, and embolic devices. The focus of this timely review is on biodegradable devices with regulatory approval in clinical trials or, at least, in advanced research stages. It also provides a comprehensive analysis of opportunities and challenges in commercialization of cardiovascular interventional implants.

The next two papers deal with a very important research challenge related to in situ (in vivo) monitoring of the evolution of degradation of biodegradable implants. Both papers focus on monitoring biodegradable Mg-based orthopedic implants that are already available commercially. In the paper entitled "Non-invasive Degradation Tracking of $\mathrm{Mg}$ Implants in Humans: A Measurement Approach," researchers from Syntellix AG in Germany, representing the first European company with CE-certified Mg-based orthopedic products on the market, 
describe noninvasive methods for detecting the progression of $\mathrm{Mg}$-based implant degradation in humans. Two methods assessed for clinical application include hydrogen gas release measurements and metal detection. The first method relies on the monitoring of hydrogen gas (which escapes from implants during $\mathrm{Mg}$ degradation through surrounding tissue and skin) production during the entire degradation time after Mg-based material implantation. In the second method, the authors used highsensitivity metal detector to monitor reduction in implant volume. Due to their simplicity, noninvasiveness, and promising accuracy and reproducibility, both methods appear suitable for a clinical setting for patients with Mg-based orthopedic implants. Also, William R. Heineman et al., in their paper entitled "Visual Hydrogen Mapping Sensor for Noninvasively Monitoring Bioresorbable Magnesium Implants In Vivo," describe a new noninvasive method for tracking the evolution of hydrogen gas produced by the implant that is monitored on the patient's skin. The authors invented a hydrogen gas sensor composed of a polymer strip with a deposited film of $\mathrm{MoO}_{3}$ and $\mathrm{Pd}$ catalytic nanoparticles which registers and shows (visually) different colors, from gray to dark blue, once exposed to different hydrogen gas concentration. This new cost-effective method could be implemented in commercially available sensing strips that could be broadly available through pharmacies and used by patients with implanted Mg-based devices on a daily basis, without visits to clinics and hospitals.

After 2 decades of intensive research on development and processing of biodegradable metallic materials, Mg-based alloys remain the most popular research target among metallurgical laboratories, especially in the area of orthopedic applications. This research sector is represented by three contributions to this collection of papers. In the paper "Magnesium Alloys for Open-Pored Bioresorbable Implants," Hans Jürgen Maier and his collaborators in Germany compare the performance of open-pored bioabsorbable implants, made of different commercially available $\mathrm{Mg}$ alloys, in terms of degradation rate, bone ingrowth behavior, biocompatibility, and resorbability of the individual alloying elements. The authors demonstrate the applicability of investment casting in design and fabrication of absorbable porous bone substitutes having the desirable pore geometry to facilitate bone ingrowth into porous implant structures. In the paper "Influence of Solution Heat Treatment on the Microstructure, Hardness and Stress Corrosion Behavior of Extruded Resoloy ${ }^{\circledR}$," Petra Maier and her collaborators in Germany report the effect of annealing treatment on the microstructure and stress corrosion properties of extruded Mg-10Dy-1Nd-1Zn- $0.2 \mathrm{Zr}$ (Resoloy ${ }^{\circledR}$ ) alloy. As shown by the authors, both microstructural characteristics and corrosion behavior of this rare earth-bearing alloy can be controlled through short-duration heat treatment that concludes with an alloy of uniform corrosion and free of cracking. Optimized microstructure consists of fine recrystallized grains with lamellar matrix LPSO phases. Ehsan Mostaed and his colleagues from Michigan Technological University prepared the paper "Tailoring the Mechanical and Degradation Performances of $\mathrm{Mg}-2.0 \mathrm{Zn}-0.5 \mathrm{Ca}-$ 0.4Mn Alloy through Microstructure Design," in which they present a novel nonequilibrium processing method for fabrication of biodegradable materials with nanostructure and improved mechanical strength. By combining rapid solidification through melt spinning and consolidation of ribbons using hot extrusion, both strengthening and enhanced corrosion resistance in $\mathrm{Mg}$ alloy was achieved, demonstrating the effective engineering of the solute nanostructures through careful control of alloy chemistry and optimizing the thermal and mechanical processing route.

Beside $\mathrm{Mg}$-based materials, both $\mathrm{Zn}$ - and $\mathrm{Fe}-$ based alloys are also widely explored for biodegradable medical implants. In the paper "Effect of Silver on the Corrosion Behavior of a Plastically Deformed Twinning-Induced Plasticity Steel for Biodegradable Stents," Diego Mantovani and his team describe their study on the combined effect of silver addition to twinning-induced plasticity steel (made of $\mathrm{Fe}, \mathrm{Mn}$, and $\mathrm{C}$ ) and its plastic deformation on steel's corrosion characteristics. The authors show that this twinning-induced plasticity steel, having excellent mechanical properties, is a very promising material for biodegradable vascular stents, in which strut diameter could be reduced to $60 \mu \mathrm{m}$ or less. Although the addition of $\mathrm{Ag}$ does not accelerate the steel's degradation, the authors found that it promotes a more uniform degradation. In the last contribution to this collection, Irsalan Cockerill et al., in their paper "Salt Preform Texturing of Absorbable Zn Substrates for Bone-Implant Applications," report on an innovative manufacturing route to tailor the surface texture of absorbable $\mathrm{Zn}$ scaffolds for orthopedic implants. The authors demonstrate a NaCl-assisted casting method in which molten $\mathrm{Zn}$ is poured over fine and coarse $\mathrm{NaCl}$ grains, which were next dissolved in water after casting solidification and cooling. The resulting fine and coarse texture $\mathrm{Zn}$ surfaces with different roughness characteristics showed accelerated corrosion rates as compared with polished $\mathrm{Zn}$ surfaces and tolerable attachment, proliferation, adhesion, and viability for mouse calvaria preosteoblast cells.

The abovementioned papers have been published under the topic of Biodegradable Materials for Medical Applications II in the following order:

- "Bio-absorbable Cardiovascular Implants: Status and Prognosis" by Subbu Venkatraman, Huang Yingying, and Wong Yee Shan.

- "Non-invasive Degradation Tracking of Mg Implants in Humans: A Measurement Approach" 
by Patrick A. Varady, Tim Vockensohn, Kristin Forßmann, Andreas Ziegler, and Jan-Marten Seitz.

- "Visual Hydrogen Mapping Sensor for Noninvasively Monitoring Bioresorbable Magnesium Implants In Vivo" by Daoli Zhao, Lingyao Wu, DaTren Chou, William Hoagland, David Benson, Zhongyun Dong, Prashant N. Kumta, and William R. Heineman.

- "Magnesium Alloys for Open-Pored Bioresorbable Implants" by Hans Jürgen Maier, Stefan Julmi, Sabine Behrens, Christian Klose, Ann-Kathrin Krüger, Peter Wriggers, AnjaChristian Waselau, and Andrea Meyer-Lindenberg.

- "Influence of Solution Heat Treatment on the Microstructure, Hardness and Stress Corrosion Behavior of Extruded Resoloy ${ }^{\circledR}$ " by P. Maier, A. Steinacker, B. Clausius, and N. Hort.

- "Tailoring the Mechanical and Degradation Performances of $\mathrm{Mg}-2.0 \mathrm{Zn}-0.5 \mathrm{Ca}-0.4 \mathrm{Mn}$ Alloy through Microstructure Design" by Ehsan Mostaed, Malgorzata Sikora-Jasinska, Lifei Wang, Ali Mostaed, Ian M. Reaney, and Jaroslaw W. Drelich.
- "Effect of Silver on the Corrosion Behavior of a Plastically Deformed Twinning-Induced Plasticity Steel for Biodegradable Stents" by Sergio Loffredo, Carlo Paternoster, Nicolas Giguère, Maurizio Vedani, and Diego Mantovani.

- "Salt Preform Texturing of Absorbable Zn Substrates for Bone-Implant Applications" by Irsalan Cockerill, Yingchao Su, Reid Bitten, Benjamin Cloarec, Samir Aouadi, Donghui Zhu, and Marcus L. Young.

To download any of these papers, follow the url $\mathrm{h}$ ttps://ink.springer.com/journal/11837/72/5/page/1 to the table of contents page for the May 2020 issue (vol. 72, no. 5).

\section{ACKNOWLEDGEMENT}

Funding to Jaroslaw W. Drelich, Malgorzata Sikora-Jasinska and Ehsan Mostaed was provided by US National Institute of Health (Grant No. 1R01HL144739-01A1).

Publisher's Note Springer Nature remains neutral with regard to jurisdictional claims in published maps and institutional affiliations. 